\title{
Focus on headache as an adverse reaction to drugs
}

\author{
Anna Ferrari - Luca Spaccapelo · Daniela Gallesi · \\ Emilio Sternieri
}

Received: 25 January 2009/Accepted: 6 May 2009/Published online: 4 June 2009

(C) Springer-Verlag 2009

\begin{abstract}
There are a large number of drugs inducing headache as an adverse reaction. Nevertheless, headaches as adverse reactions to drugs have received limited attention. Non-serious adverse reactions, such as headache, are not quantified and described as accurately as serious, life threatening ones. However, non-serious reactions can also be extremely troublesome, above all when they are chronic: they can affect patients' quality of life and contribute to non-compliance. It is absolutely possible that the number of patients with headache as an adverse reaction, which is going to increase, considering the growing use of medications. Physicians should, therefore, be aware of this issue. Indeed, it is difficult to attribute the diagnosis of adverse drug reaction to a condition, headache, which is also a very common symptom in general population.
\end{abstract}

Keywords Adverse drug reaction - Classification . Pharmacovigilance $\cdot$ Headache $\cdot$ Migraine $\cdot$ Side effect

\section{Introduction}

Headaches as adverse reactions to drugs have not been much studied both in the field of pharmacovigilance, and by headache clinicians and researchers, even if a very large number of drugs, with different mechanisms of action, can induce headache in acute or chronic treatment or after drug discontinuation [1]. The aims of this paper are (1)

\footnotetext{
A. Ferrari $(\bowtie) \cdot$ L. Spaccapelo $\cdot$ D. Gallesi · E. Sternieri Headache Centre, Division of Toxicology and Clinical Pharmacology, University Centre for Adaptive Disorders and Headache, Section Modena II. University of Modena and Reggio Emilia, Policlinico, Largo del Pozzo, 71, 41100 Modena, Italy e-mail: anna.ferrari@unimore.it
}

underscoring the lack of information on headache as an adverse reaction, and (2) stimulate to recognize the kind of adverse reaction headache, in order to properly treat it.

\section{Adverse reactions}

Medications are classified according to their main therapeutic action, but no drug induces only one specific effect. Drugs can be selective, but they are rarely specific. They induce two kinds of effects: those useful for treatment and those non-relevant to this aim and unintended. The latter are defined "side effects" or, more specifically, adverse reactions. An adverse drug reaction (ADR) is a response to a drug that is noxious and unintended and occurs at doses normally used in people for the prophylaxis, diagnosis or therapy for the disease, or for modification of physiological function [2]. Adverse reactions are classified into serious and non-serious or low-risk reactions. A serious reaction is one that results in death, requires hospital admission or prolongation of existing hospital stay, results in persistent or significant disability/incapacity, or is life threatening [3].

Adverse reactions are categorized into five types, the most important of which are type-A (augmented), dosedependent, and type-B (bizarre), non-dose-dependent [4]. Most adverse reactions, approximately 75\% [5], belong to type-A [6]. These ADRs are relatively easy to diagnose, since they can be predicted according to the main action of the drug and are, therefore, generally identified in the study preceding the marketing of the drug. They have high morbidity, but are practically never life threatening. Furthermore, they can disappear if drug dosage is reduced [6]. Type-B reactions are also termed idiosyncratic, owing to their unpredictable nature and our lack of understanding of the mechanisms involved. There is no dose-response 
relationship, incidence is very low $(<1 / 1,000)$, but they are often serious and potentially life threatening. They are often not detected until the drug has been released onto the market and they are sometimes detected even after years of use. Type-B reactions, therefore, represent a major clinical problem [7]. Type-C reactions (arising from chronic use) are induced by the long-term effect of the drug and its metabolites: for example, the long-term toxicity of amiodarone. Type-D (delayed) is adverse reactions that appear later and are more serious than type-C: for example, bladder cancer in patients who had been treated for a long period with cyclophosphamide. Type-E reactions (end of treatment) arise from discontinuing treatment [8].

The identification of ADRs has remarkably improved in the last decades [9]. Nevertheless, reactions that are not life threatening are not systematically studied and quantified with the same accuracy as those which are considered serious [10]. Serious events are five times more likely to be reported than non-serious ones [11, 12]. Even when they are reported, non-serious ADRs are often not described in detail, unless the event is a study endpoint or it is of particular interest. This is unfortunate, because non-serious ADRs can be very worrying [13], above all when they are chronic: they can affect patients' quality of life, reduce adherence to treatment or cause drug discontinuation $[10,14,15]$.

There are various information sources about ADRs. The most important ones are randomized controlled trials, epidemiological post-marketing studies, and spontaneous reporting [16]. ADR headache is very frequent, whatever the source is. However, information about this ADR is usually limited and does not allow a precise assessment. Clinical trials should, therefore, be able to determine ADR headache more accurately; above all, when the drug studied is related to a previously marketed compound able to induce headache, or its mechanism of action can supposedly cause headache. Moreover, when the results of clinical trials are published, the proportion of patients that discontinued drug treatment as a consequence of headache should also be reported together with a detailed description of the characteristics of the symptom and of the patients.

The actual frequency of an ADR headache is only known when a drug has been studied both in patients taking it and in placebo group. The importance of this datum is also stressed in ICHD-II [17], which specifies in chapter 8.1 that "only when headache occurs more often after active drug than after placebo in double blind trials it can be regarded as true effect". Nevertheless, the placebo effect can substantially vary across trials with the same drug [18]. The frequency of ADR headache can also be influenced by the presence of headache sufferers, who could be much more susceptible to this adverse reaction than non-headache subjects [17]. Patients should therefore be screened to detect headache before clinical trial enrolment and headache sufferers should be separately assessed.

\section{ADR headache}

ICDH-II [17] includes secondary headaches attributed to drugs or their withdrawal, which can be fully considered adverse reactions to drugs, in chapter 8 , codes $8.1,8.3$, and 8.4. Many other ADR headaches have also been less studied and characterized that in lacking elements for a more accurate description, ICHD-II itself [17] only gives a list of the drugs, which can induce them in the appendix.

The two mechanisms inducing headache as an adverse reaction have been classically considered: one is vasodilatation and the other is raised intracranial pressure. Vasodilatation explains headache induced by most cardiovascular drugs such as calcium-channel blockers and ACE inhibitors. In these cases, headache is a side effect related to the primary pharmacological action of the drug, it is often dose-dependent, but it already appears with the dosages normally used in therapy. Headache related to aseptic meningitis induced by drugs is instead a type-B adverse reaction, not predictable from the mechanism of action of the medication that induces it [1].

Many ADR headaches can have the characteristics of migraine, tension-type headache or cluster headache (Table 1) and could therefore contribute to the high individual and social cost of these diseases. The burden associated with primary headache is a major public health problem [19]. In a retrospective study carried out in the Netherlands [20], headache was the third ADR most frequently reported by patients and even the first reported by

Table 1 Characteristics of headaches attributed to drugs and drug classes

\begin{tabular}{ll}
\hline Characteristic & Drug \\
\hline Migraine without aura & Cyclosporin [31], Dipyridamole [32], Nitric oxide donors [33], Phosphodiesterase inhibitors [34], \\
& Interferon- $\beta$ [35], Ondansetron [36], Tacrolimus [37], Sertraline [38] \\
Migraine with aura & Nitric oxide donors [39], Phosphodiesterase inhibitors [34], Tacrolimus [40], Fluoxetine [41] \\
$\begin{array}{l}\text { Typical aura without headache } \\
\text { Cluster headache }\end{array}$ & Tadalafil [42] \\
\hline
\end{tabular}


health-care professionals in the year 2004-2007. Patients only report reactions which they consider important; they do not instead report the ones which they consider nonserious, unless they are specifically questioned (which has not been done in this study). These results therefore indicate that, even if it was considered non-serious, headache was very important, both for patients and for health-care professionals. There are no standardized and universal rating scales to categorize the severity of ADR. There is no single risk-benefit evaluation for a drug; it is contextdependent [21]. For example, the risk of headache induced by an immunosuppressant treatment is less important than the benefit, whereas it becomes more relevant in cases that only require anti-acid therapy.

Considering the growing number of people taking drugs [22], headache clinicians are likely to treat patients with polymedication and headaches as ADRs, difficult to diagnose and manage. In this context, every time a patient reports the onset of a headache he had never suffered from or the worsening of or change in the headache he has been suffering from for a long time, the physician shall also take into consideration, in differential diagnosis, that this headache can be an adverse reaction to a drug. An accurate pharmacological anamnesis is fundamental in order to diagnose headache as an adverse reaction. Nevertheless, it can be very difficult to attribute the diagnosis of ADR to a condition such as headache, which is also a common symptom, not pathognomonic of any pathological condition, and extremely widespread among general population (the percentage of the adult population with an active headache disorder is $47 \%$ for headache in general) [23]. Primary headaches, such as ADRs, are prevalent in females [24]. In clinical practice, the consequences of this fact are probably reduced because episodic primary headaches mainly affect young adults who take few medications [23]. However, a large number of chronic headache patients attend a tertiary clinic and the majority of them are elderly women with multiple co-morbid conditions and polypharmacy $[24,25]$, and therefore are at a higher risk of ADRs [26].

In order to define the causal relationship between drug and adverse reaction, the time elapsed between the onset of headache and the administration of the drug must be considered and it must be assessed if the headache is compatible with the well-known pharmacological properties of the substance. However, the best proofs of the causal relationship are the disappearing of headache after discontinuing the drug and its onset when the patient takes the medication again [27].

Failing to detect ADR headache can cause patients' suffering, anxiety, worrying, and a high social cost: diagnostic tests, other physicians' consultations, and prescription of more drugs, while the solution is exactly the opposite, i.e., to reduce the number of drugs [28].

Researchers should also be interested in ADR headache, considering the fundamental role that drugs have had and will have in deepening the knowledge of pathogenetic mechanisms of primary headaches. An example is NO donors. These drugs have been widely used in experimental models and diagnostic tests to study headaches [29].

Table 2 Classification of ADR headaches according to the type of adverse reaction (drug-induced headaches reported in ICHD-II have not been included when there are no elements proving or suggesting the causal link between drug and headache)

Headache type Drugs implied

1. Headache as type-A adverse drug reaction: predictable, related to the principal pharmacological action of the drugs, and dosedependent

2. Headache as type-B adverse drug reaction: idiosyncratic, unpredictable, and related to aseptic meningitis (code 7.3.2)

3. Headache as type-C adverse drug reaction: after chronic medication, related to raised intracranial pressure (code 8.3 and code 7.1.2)

4. Headache as type-E adverse drug reaction: related to substance withdrawal
Nitric oxide (NO) donors (code 8.1.1), Phosphodiesterase (PDE) inhibitors (code 8.1.2), Calcitonin gene-related peptide-CGRP (code 8.1.9), Cocaine (code 8.1.6), Ethanol (code 8.1.4), Cannabis (code 8.1.7), Histamine (code 8.1.8), Calcium-channel blockers (Table 1 in the appendix), Antiarrhytmics, $\beta$-Adrenergic blockers, Ace inhibitors, Sympathomimetics (code 10.3.6), Antagonists of the at-1 receptors for Angiotensin II, Statins, $\alpha_{2}$-Adrenergic agonist (Clonidine), $\alpha_{1}$-Adrenergic blockers (Doxazosin and Prazosin), Amiloride, Methylxanthines, $\beta_{2}$-Adrenergic agonists, Agents for erectile dysfunction, Ergotamine (Table 1 in the appendix), Nicotine (code 8.1.10), Amphetamine (code 10.3.6)

Amoxicillin, Carbamazepine, Diclofenac, Famotidine, Ibuprofen, Immune globulin, Infliximab, Ketorolac, Leflunomide, Levamisole, Metronidazole, Naproxen, Ranitidine, Rofecoxib, Sulfamethoxazole, Sulfasalazine, Sulindac, Tolmetin, Trimethoprim, Valacyclovir

Amiodarone, Anabolic steroids, Contraceptives Combination, Ciprofloxacin, Danazol, Corticosteroids, Gentamicin, Lithium Carbonate, Nalidixic Acid, Nitrofurantoin, Ofloxacin, Retinoic Acid, Tetracycline, Thyroid hormone replacement, Vitamin A

Caffeine-withdrawal headache (code 8.4.1), Opioid-withdrawal headache (code 8.4.2), estrogen-withdrawal headache (code 8.4.3), Ergotamine-withdrawal headache, Cocaine-withdrawal headache, Methysergide-withdrawal headache

* The codes reported in ICHD-II are in brackets 
In order to give more elements to recognize and treat adverse reaction headaches, drug-induced headaches [excluding medication-overuse headache (code 8.2)] included in chapter 8 of the International Classification of Headache Disorders (ICHD-II) could also be classified according to the criteria and terminology used to categorize adverse reactions in the field of pharmacovigilance (Table 2). This classification stresses the pathophysiological basis of these "headaches induced by drug use", an essential element to classify them as secondary. Furthermore, it suggests immediately the possible management of type-A adverse reaction headaches, that is to say, the reduction of drug dosage. This classification has some limitations, due to the uncertainty inherent in the different sources. However, we excluded drugs inducing percentages of headache lower than those induced by placebo. It should also be stressed in Table 2 that ADR headaches which clearly show the causal link between drug and headache are those classified into $\mathrm{A}$ and $\mathrm{E}$ types. The hypothesis of causal relationship is often only formulated considering the evolution of case reports in type-C headaches.

Classifications are very important to organize and manage knowledge and establish relationships between the various pieces of information. The classification of the International Headache Society has become a diffused standard in World wide communications between researchers and clinicians. The methods used to analyze and assess drug safety are more and more precise [30]. In this context, we also propose that clinical trials also assess if the side effect headache has or has not characteristics resembling migraine, tension-type headache or cluster headache. This effort should allow accurate descriptions of the headache's characteristics before drug approval and it should make the diagnosis of ADR headache easier in clinical practice after the drug is marketed.

We hope that the sharing of the classification used in the field of pharmaco-vigilance and headaches will improve the management of patients' problems.

\section{Conflict of interest None.}

\section{References}

1. Ferrari A (2006) Headache: one of the most common and troublesome adverse reactions to drugs. Curr Drug Saf 1:43-58

2. World Health Organization (1969) International drug monitoring: the role of the hospital. Tech Rep Ser 425:5-25

3. World Health Organization (1981) Cancer treatment: WHO recommendations for grading of acute and subacute toxicity. Cancer 47:207-214

4. Kessler DA (1993) MEDWatch: the new FDA medical products reporting program. Clin Pharm 12:529-532

5. Cohen JS (2002) Do standard doses of frequently prescribed drugs cause preventable adverse effects in women? J Am Med Womens Assoc 57:105-110
6. Park BK, Pirmohamed M, Kitteringham NR (1992) Idiosyncratic drug reactions: a mechanistic evaluation of risk factors. Br J Clin Pharmacol 34:377-395

7. Ju C, Uetrecht JP (2002) Mechanism of idiosyncratic drug reactions: reactive metabolite formation, protein binding and the regulation of the immune system. Curr Drug Metab 3:367-377

8. Nebeker JR, Barach P, Samore MH (2004) Clarifying adverse drug events: a clinician's guide to terminology, documentation, and reporting. Ann Intern Med 140:795-801

9. Hartford CG, Petchel KS, Mickail H et al (2006) Pharmacovigilance during the pre-approval phases: an evolving pharmaceutical industry model in response to ICH E2E, CIOMS VI, FDA and EMEA/CHMP risk-management guidelines. Drug Saf 29:657-673

10. Michels KB (1999) Problems assessing non-serious adverse drug reactions: antidepressant drug therapy and sexual dysfunction. Pharmacotherapy 19:424-429

11. Heeley E, Riley J, Layton D et al (2001) Prescription-event monitoring and reporting of adverse drug reactions. Lancet 358:1872-1873

12. Ioannidis JPA, Lau J (2002) Improving safety reporting from randomised trials. Drug Saf 25:77-84

13. Neidig JL, Koletar SL (2001) Safety reporting in clinical trials. J Am Med Assoc 285:2077-2078

14. Kaufman DW, Shapiro S (2002) Epidemiological assessment of drug-induced disease. Lancet 356:1339-1343

15. Hazell L, Shakir SA (2006) Under-reporting of adverse drug reactions : a systematic review. Drug Saf 29:385-396

16. Venulet J, ten Ham M (1996) Methods for monitoring and documenting adverse drug reactions. Int J Clin Pharmacol Ther 34:112-129

17. Headache Classification Subcommittee of the International Headache Society (2004) The International Classification of Headache Disorders, 2nd edn. Cephalalgia 24(Suppl 1):1-160

18. Rief W, Avorn J, Barsky AJ (2006) Medication-attributed adverse effects in placebo groups: implications for assessment of adverse effects. Arch Intern Med 166:155-160

19. Hazard E, Munakata J, Bigal ME et al (2009) The burden of migraine in the United States: current and emerging perspectives on disease management and economic analysis. Value Health 12:55-64

20. De Langen J, van Hunsel F, Passier A et al (2008) Adverse drug reaction reporting by patients in the Netherlands: three years of experience. Drug Saf 31:515-524

21. Boada JN, Boada C, García-Sáiz M et al (2008) Net efficacy adjusted for risk (NEAR): a simple procedure for measuring risk: benefit balance. PLoS ONE. doi:10.1371/journal.pone. 0003580

22. Veehof LJ, Stewart RE, Meyboom-de Jong B et al (1999) Adverse drug reactions and polypharmacy in the elderly in general practice. Eur J Clin Pharmacol 55:533-536

23. Jensen R, Stovner LJ (2008) Epidemiology and comorbidity of headache. Lancet Neurol 7:354-361

24. Zopf Y, Rabe C, Neubert A et al (2008) Women encounter ADRs more often than do men. Eur J Clin Pharmacol 64:999-1004

25. Ferrari A, Leone S, Vergoni AV et al (2007) Similarities and differences between chronic migraine and episodic migraine. Headache 47:65-72

26. Franceschi M, Scarcelli C, Niro V et al (2008) Prevalence, clinical features and avoidability of adverse drug reactions as cause of admission to a geriatric unit: a prospective study of 1756 patients. Drug Saf 31:545-556

27. Edwards IR, Aronson JK (2000) Adverse drug reactions: definitions, diagnosis, and management. Lancet 356:1255-1259

28. White TJ, Arakelian A, Rho JP (1999) Counting the costs of drug-related adverse events. Pharmacoeconomics 15:445-458 
29. Iversen HK, Olesen J (1994) Nitroglycerin-induced headache is not dependent on histamine release: support for a direct nociceptive action of nitric oxide. Cephalalgia 14:437-442

30. Ross SD (2001) Drug-related adverse events: a readers' guide to assessing literature reviews and meta-analyses. Arch Intern Med 161:1041-1046

31. Steiger MJ, Farrah T, Rolles K et al (1994) Cyclosporin associated headache. J Neurol Neurosurg Psychiatry 57:1258-1259

32. Kruuse C, Lassen LH, Iversen HK et al (2006) Dipyridamole may induce migraine in patients with migraine without aura. Cephalalgia 26:925-933

33. Thomsen LL, Kruuse C, Iversen HK et al (1994) A nitric oxide donor (nitroglycerin) triggers genuine migraine attacks. Eur $\mathrm{J}$ Neurol 1:73-80

34. Evans RW, Kruuse C (2004) Phosphodiesterase-5 inhibitors and migraine. Headache 44:925-926

35. Khromov A, Segal M, Nissinoff J et al (2005) Migraines linked to interferon-beta treatment of multiple sclerosis. Am J Phys Med Rehabil 84:644-647

36. Khan RB (2002) Migraine-type headaches in children receiving chemotherapy and ondansetron. J Child Neurol 17:857-858
37. Ferrari U, Empl M, Kim KS et al (2005) Calcineurin inhibitorinduced headache: clinical characteristics and possible mechanisms. Headache 45:211-214

38. Munera PA, Goldstein A (2001) Migraine and sertraline. J Am Acad Child Adolesc Psychiatry 40:1125-1126

39. Bank J (2001) Migraine with aura after administration of sublingual nitroglycerin tablets. Headache 41:84-87

40. Toth CC, Burak K, Becker W (2005) Recurrence of migraine with aura due to tacrolimus therapy in a liver transplant recipient successfully treated with sirolimus substitution. Headache 45:245-246

41. Larson EW (1993) Migraine with typical aura associated with fluoxetine therapy: case report. J Clin Psychiatry 54:235-236

42. Dinn RB, Wall M (2006) Tadalafil associated with typical migraine aura without headache. Cephalalgia 26:1344-1346

43. Ekbom K (1968) Nitrolglycerin as a provocative agent in cluster headache. Arch Neurol 19:487-493

44. Evans RW (2006) Sildenafil can trigger cluster headaches. Headache 46:173-174 